\title{
Ondes internes \\ à l'interface de deux fluides de densités voisines contenus dans un bassin rectangulaire tournant
}

\author{
par G. Chabert-d'Hières \\ Ingénieur au C.N.R.S., \\ détaché à l'Institut de Mécanique \\ de Grenoble
}

\author{
ef J.-L. Suberville \\ Ingénieur \\ à I'Institut de Mécanique \\ de Grenoble
}

\section{I - Position du problème}

On se propose d'étudier les oscillations baroclines d'une bicouche formée de deux liquides superposés de densités différentes, contenus dans une cuve rectangulaire tournant à une vitesse uniforme, et de profondeur constante.

A partir d'une théorie de Taylor, le problème a été mis en équation (cf. [1]). Nous avons récemment explicité des solutions analytiques linéaires à un ordre d'approximation suffisant pour les applications pratiques.

Le contrôle expérimental a été réalisé dans une cuve implantée sur la plaque tournante de l'Université de Grenoble. Après avoir décrit sommairement l'installation d'essais, on montre l'influence de la rotation sur la période propre de l'onde d'interface (le rôle de la diffusion est mis en évidence), ainsi que sur la configuration (réseaux d'égales amplitudes et d'égales phases) de l'interface; les effets non-linéaires, ceux dûs aux dimensions finies du modèle sont discutés.

\section{II - Théorie}

\section{II.1 - Notations}

Soit $O x y z$ un repère orthonormé (l'axe $O z$ est vertical) animé, par rapport à un repère fixe, d'un mouvement de rotation uniforme, de vitesse angulaire $\omega$, autour d'une verticale fixe. Une cuve contient deux liquides de masse volumique $\rho_{i},\left(\Delta \rho=p_{2}-p_{1}>0\right)$ (on affectera des indices 1 et 2 les caractéristiques des liquides supérieur et inférieur, respectivement), occupant, au repos, les domaines $o \leqslant x \leqslant a, \quad o \leqslant y \leqslant b, \quad o \leqslant z \leqslant h_{1}, \quad$ et $o \leqslant x \leqslant a$, $o \leqslant y \leqslant b-h_{2} \leqslant z \leqslant o$ (cf. figure 1), $U_{x_{i}}(x, y, z, t)$, $U_{y_{i}}(x, y, z, t)$ et $U_{z_{i}}(x, y, z, t)$ sont les composantes suivant Oxy $z$ de la vitesse $\vec{V}_{i}(x, y, z, t)$. On note $P(x, y, z, t)$ et $p(x, y, z, t)$ respectivement le potentiel total et la pression; $\varepsilon(x, y, z, t)$ désigne le soulèvement vertical du point de coordonnées $(x, y, z)$ à l'état d'équilibre; $g$ l'accélération de la pesanteur; $\Sigma_{1}$ désigne la surface libre, et $\varepsilon_{1}\left(x, y, h_{1} t\right)$ sa dénivellation par rapport au plan $z=h_{1} ; \Sigma_{2}$ l'interface et $\varepsilon_{2}(x, y, o, t)$ sa dénivellation par rapport au plan $z=o$.

\section{II.2 - Hypothèses}

On néglige les tensions superficielles sur $\Sigma_{i}$, la viscosité des liquides, la force centrifuge; les équations de l'hydro-

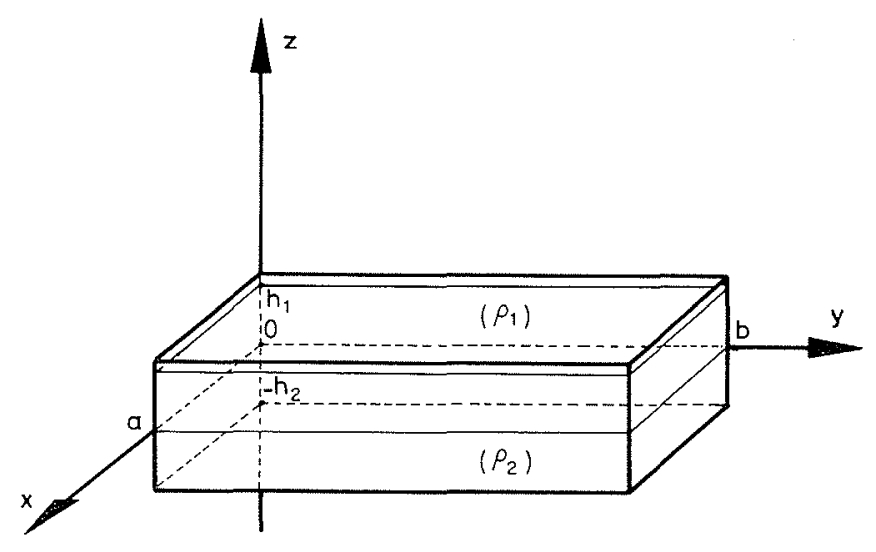

1/ Notations. 
dynamique sont linéarisées: $\varepsilon_{1}$ et $\varepsilon_{2}$ sont supposées petites vis-à-vis de $h_{1}$ et $h_{2}$. On se limite à l'étude des oscillations libres harmoniques et on suppose toutes les grandeurs $U_{x}$ $U_{y_{i}}, U_{z_{i}}, P_{i}, p_{i}, \varepsilon_{i}$ égales respectivement au produit de fonctions de $x, y, z$ par $e^{i \theta t}$. Contrairement aux théories habituelles semi-dynamiques, on ne néglige pas la composante verticale des accélérations.

\section{II.3 - Equations fondamentales}

Les équations linéarisées, homogènes du mouvement de chaque liquide s'écrivent (cf. [1]):

(1) $\left\{\begin{array}{l}\overrightarrow{\partial V_{i}} / \partial t+2 \vec{\omega} \wedge \vec{V}_{i}=\overrightarrow{\operatorname{grad}} P_{i} \\ \operatorname{div} \vec{V}_{i}=0\end{array}\right.$

$$
\begin{aligned}
& P_{i}=-\frac{p_{i}}{P_{i}}-g\left(z+\varepsilon_{i}\right) \\
& U_{z_{i}}=\partial \varepsilon_{i} / \partial t
\end{aligned}
$$

On montre que l'on a, pour les déplacements verticaux $\varepsilon$, compte tenu des hypothèses, et en posant

$$
\varepsilon_{i}=\varepsilon_{x y_{i}} \cdot \varepsilon_{z_{i}}
$$

les équations suivantes :

$$
\left\{\begin{array}{l}
\Delta \varepsilon_{x y_{i}}+q^{2} \frac{\theta^{2}-4 \omega^{2}}{\theta^{2}} \varepsilon_{x y_{i}}=0 \\
\partial^{2} \varepsilon_{z_{i}} / \partial t^{2}=q^{2}
\end{array}\right.
$$

où $q$, paramètre issu de la séparation des variables, est homogène à un nombre d'onde.

Intéressons-nous au cas où $q^{*}$ est réel, ce qui correspond aux expériences que nous avons réalisées, et recherchons les solutions de (1) sous la forme

(4)

$$
\left\{\begin{array}{l}
P_{i}=e^{i \theta t} \cdot \operatorname{chq}\left(z+z_{o_{i}}\right) \cdot \pi_{i}(x, y) \\
U_{x_{i}}=e^{i \theta t} \cdot \operatorname{chq}\left(z+z_{o_{i}}\right) \cdot u_{i}(x, y) \\
U_{y_{i}}=e^{i \theta t} \cdot \operatorname{chq}\left(z+z_{o_{i}}\right) \cdot v_{i}(x, y) \\
U_{z_{i}}=e^{i \theta t} \cdot \operatorname{shq}\left(z+z_{o_{i}}\right) \cdot w_{i}(x, y)
\end{array}\right.
$$

\section{Expression des conditions aux limites}

La nullité de la vitesse verticale sur le fond est automatiquement vérifiéc, pourvu que $z_{0,3}=h_{2 .}$. Le fait que $\Sigma_{1}$ est à pression constante se traduit à partir de (4) par la relation transcendante

$$
\theta^{2}=g q \text { thq }\left(h_{1}+z_{o_{1}}\right)
$$

En éliminant $z_{0_{1}}$ entre (5) et les relations exprimant l'égalité des $U_{z_{i}}$ et des $P_{i}$ à l'interface, on obtient la relation de dispersion:
$(6$

$$
\begin{aligned}
& \theta^{4}\left(1+\frac{p_{1}}{P_{2}} t_{1} t_{2}\right)-\theta^{2} g q\left(t_{1}+t_{2}\right)+g^{2} q^{2} \frac{\Delta p}{p_{2}} t_{1} t_{2}=0 \\
& t_{1}=\text { thqh, } \quad t_{2}=\text { thqh }
\end{aligned}
$$

On montre, lorsque $\Delta \rho / \rho_{2}$ est suffisamment petit, ce qui est le cas lors de nos expériences, que (6) se scinde en

$$
\begin{aligned}
& \theta^{2}=g \frac{\Delta p}{p_{2}} q \frac{t_{1}+t_{2}}{p_{1} t_{2}+p_{2} t_{1}} \\
& \theta^{2}=g q \operatorname{th} q\left(h_{1}+h_{2}\right)
\end{aligned}
$$

On reconnaît les expressions de la pulsation relative correspondant, respectivement, au mode interne (ou barocline) qui nous intéresse, et au mode externe (ou barotrope).

Les conditions aux parois verticales se notent

$$
\left\{\begin{array}{l}
u_{i}(x, y)=0 \text { pour } x=0 \text { et } a \\
v_{i}(x, y)=0 \text { pour } y=0 \text { et } b
\end{array}\right.
$$

et se traduisent par les expressions:

$$
\begin{cases}i \theta \frac{\partial \varepsilon_{x y_{i}}}{\partial x}+2 \omega \frac{\partial \varepsilon_{x y_{i}}}{\partial y}=0 & \text { pour } x=0 \text { et } a . \\ i \theta \frac{\partial \varepsilon_{x y_{i}}}{\partial y}-2 \omega \frac{\partial \varepsilon_{x y_{i}}}{\partial x}=0 & \text { pour } y=0 \text { et } b .\end{cases}
$$

\section{II.4 - Intégration dans un bassin rectangulaire} (méthode des fonctions propres)

Le problème consiste à rechercher une fonction $\varepsilon_{x: y,}$ vérifiant (3) et astreinte aux conditions (7) et (9). Il se trouve que nous retrouvons là, formellement, le problème que celui que G. I. Taylor, [2], a résolu dans le cas d'un bassin rectangulaire tournant contenant une couche liquide homogène de faible profondeur (vitesse verticale négligée). Nous avons étendu son procédé à notre cas en recherchant, comme nous l'a suggéré le Professeur Kravtchenko, la fonction $\varepsilon_{x y_{1}}$, sous forme d'une série généralisée de Fourier, dont (9), compte tenu de (7) permet de déterminer les coefficients. On est conduit à une équation $D_{\infty}(\theta, q)=0$ qui, combinée avec l'équation de dispersion, fournit l'équation aux pulsations propres; $D_{\%}$ prend la forme d'un déterminant infini pour lequel les critères de convergence ne sappliquent pas. Comme Taylor, nous nous sommes limités à des procédés approchés.

\section{Période propre}

En se limitant à l'ordre 2, on obtient une formule prenant une expression relativement simple, permettant de calculer la période propre de la seiche d'interface en fonction de la vitesse de rotation. Lapplication numérique correspondant à nos expériences montre que les résultats graphiques obtenus à l'aide de cette formule ne diffèrent pas de ceux obtenus par Rao [3], à l'aide d'une autre théorie (à l'ordre 20). Il s'agit, pour $Y$ voisin de 1, de : 


$$
\left\{\begin{array}{l}
Y \operatorname{tg} \frac{\pi}{2} Y=\frac{x^{2}+b^{2} / a^{2} \pi}{x^{3}} \cdot \frac{\pi}{4} \sqrt{x^{2}-1+b^{2} / a^{2}} \cdot \text { th } \frac{\pi}{2} \sqrt{x^{2}-1+b^{2} / a^{2}} \cdot \frac{\operatorname{th} \pi a x / 2 b}{a / b} \\
\text { avec } \quad X=2 \omega / \theta_{0} \quad Y=\theta / \theta_{0} \quad\left(\theta_{0}=\theta \text { pour } \omega=0\right)
\end{array}\right.
$$

D’autre part, on a résolu numériquement l'équation aux pulsations propres à l'ordre $2,3, \ldots 10$. Les résultats montrent que la convergence des solutions est extrêmement rapide.

\section{Configuration de l'onde}

Un calcul laborieux permet d'obtenir les coefficients du développement en série de la fonction $\varepsilon_{i r} y_{\mathfrak{i}}$ pour des ordres élevés. Néanmoins, la convergence étant très rapide on peut se limiter à l'ordre 4, dans les applications numériques.

Comme pour la période propre, on peut montrer qu'un développement à l'ordre 2 , seulement, donne de bons résultats (il s'agit alors de la superposition d'une amphidromie de Kelvin et d'une seule onde de Poincaré (de $2^{\prime \prime}$ espèce). Le soulèvement $\widetilde{\varepsilon}_{2,}$ de l'interface s'exprime alors sous la forme suivante :

$$
\begin{aligned}
& \text { (11) }\left\{\begin{array}{l}
\tilde{\varepsilon}_{2}=\varepsilon^{\prime} \cos \theta t-\varepsilon^{\prime \prime} \sin \theta t \\
\varepsilon^{\prime}=-Y_{0} \sin q\left(y-\frac{b}{2}\right) \operatorname{ch} \frac{q}{\theta} 2 \omega\left(x-\frac{a}{2}\right)-\cos \beta \cdot \operatorname{sh} \alpha \cdot \frac{Y_{1}}{\operatorname{ch} \beta_{1}} \frac{a}{b} \frac{2 \omega}{\theta} \frac{L_{1}}{r} \operatorname{sh} \frac{L_{1}}{b}\left(y-\frac{b}{2}\right) \cdot \sin \pi \frac{x}{a} \\
\varepsilon^{\prime \prime}=-Y_{0} \cos q\left(y-\frac{b}{2}\right) \operatorname{sh} \frac{q}{\theta} 2 \omega\left(x-\frac{a}{2}\right)+\cos \beta \cdot \operatorname{sh} \alpha \cdot \frac{Y_{1}}{\operatorname{ch} \beta_{1}} \operatorname{ch} \frac{L_{1}}{b}\left(y-\frac{b}{2}\right) \cdot \cos \pi \frac{x}{a}
\end{array}\right. \\
& \text { avec } \quad \begin{array}{l}
\frac{Y_{0}}{Y_{1}}=\frac{\frac{9}{5 K^{2}}-\frac{3 S_{2}}{1+K^{2}}-\frac{1}{4+K^{2}}}{\frac{32}{15}+3 S_{1} S_{2}} \\
K=2 \omega q a / \pi \theta, \quad, \quad Y_{0} \text { arbitraire }
\end{array}, \\
& S_{1}=-\frac{\pi^{2}}{1 \sigma_{\alpha} \beta} L_{1} \text { th } \beta_{1} \cdot \operatorname{cotg} \beta \cdot \text { th } \alpha \text {. } \\
& S_{2}=\frac{\pi^{2}}{16 \alpha \beta} L_{2} \operatorname{coth} \beta_{2} \cdot \operatorname{tg} \beta \cdot \operatorname{coth} \alpha \\
& L_{n}=\frac{b}{a} \sqrt{n^{2} \pi^{2}-q^{2} a^{2} \frac{\theta^{2}-4 \omega^{2}}{\theta^{2}}} \quad(n=1 \text { et } 2) .
\end{aligned}
$$

Ces notations (sauf (11)) sont empruntées à Bonnefille [4], qui a adapté la méthode de Taylor à la recherche des fonctions propres du système.

\section{II.5 - Application numérique}

Des calculs de périodes propres et de profils d'onde ont été réalisés pour 5 vitesses de rotation différentes: $\omega=0$, $\omega=0,0600, \omega=0,0865\left(\theta^{2}=4 \omega^{2}\right), \omega=0,1247, \omega=0,1600 \mathrm{~s}^{-1} ;$ pour $a=0,50 \mathrm{~m}, b=2,03 \mathrm{~m}, h_{1}=h_{2}=0,15 \mathrm{~m}$, $\rho_{1}=0,99895 \mathrm{~g} / \mathrm{cm}^{3}$ et $\rho_{2}=1,01745 \mathrm{~g} / \mathrm{cm}^{3}$ (cf. tableau de la figure 8). Les résultats théoriques sont exposés dans le $\S$ IV et comparés avec les résultats expérimentaux.

\section{III - Description de l'ensemble expérimental}

La description détaillée de l'ensemble expérimental fera l'objet d'une prochaine publication. Aussi, nous bornerons-nous à donner les principes généraux de fonctionnement de l'installation. On peut distinguer trois sous-ensembles:

- Le sous-ensemble mécanique (cf. fig. 2). Celui-ci comprend le canal oscillant proprement dit dont le mouvement est commandé par un groupe moto-réducteur à vitesse variable régulée, et la plaque tournante $(300 \mathrm{t}, \varnothing 14 \mathrm{~m}$ ) sur laquelle il repose ainsi que tout le système expérimental.

- Le sous-ensemble hydraulique, constitué par une installation de dégazage d'eau de la ville, de préparation de solu- 
tions de $\mathrm{NaCl}$ dans celle-ci, et de remplissage du canal, en vue de la réalisation de systèmes à une ou plusieurs couches liquides superposées de densités différentes.

- Le sous-ensemble «appareils de mesure», utilisé pour le contrôle ou l'enregistrement des températures, pression, densités, vitesses et amplitudes verticales des ondes.

\section{Réalisation des bicouches}

Après avoir mis en rotation le système, à la vitesse choisie pour l'expérience, on introduit dans la cuve la quantité d'eau douce adéquate $\left(p_{1}, h_{1}\right)$; dès l'établissement du repos de celle-ci, le canal étant incliné du côté de l'orifice d'alimentation (constitué par une fente transversale), on injecte la solution salée $\left(\rho_{2}, h_{2}\right)$ qui pénètre lentement sous la couche d'eau douce (cf. fig. 3). Pour éviter que la diffusion résultant de l'avance du coin salé soit trop importante, la vitesse de remplissage, notamment en son début, est lente. Le remplissage dure environ une heure. On colore la couche d'eau salée, de façon à pouvoir suivre son évolution.

\section{Génération des ondes d'interface}

Les ondes sont engendrées à la résonance, en faisant osciller autour de son axe transversal médian, tangent à l'interface en son milieu, la cuve, dont une extrémité est animée d'un mouvement oscillatoire sinusoïdal. Le fréquence doit être égale à la fréquence propre de l'onde. Nous devons donc connaître et mesurer avec précision cette dernière. L'amplitude de l'excitation, généralement de l'ordre du millimètre (cf. figure 2), permet d'obtenir des ondes de plusieurs centimètres d'amplitude; habituellement l'amplification est de l'ordre de 15.

\section{Les suiveurs d'interface}

Les mesures d'amplitude sont réalisées au moyen de trois "suiveurs d'interface», appareils équipés de sondes de conductimétrie à aspiration continue, guidées verticalement, dont l'extrémité inférieure est astreinte, par un asservissement (comparaison avec une conductivité affichée), à suivre le mouvement vertical d'une couche de conductivité donnée, au sein de l'interface. L'enregistrement du mouvement est digitalisé de façon à permettre des dépouillements et des analyses précises.

\section{IV - Résultats expérimentaux Comparaison avec la théorie}

\section{IV.1 - Période propre}

Les périodes propres s'obtiennent en fixant une sonde de l'appareil précédent, de telle façon que son extrémité inférieure aspire, en l'absence d'onde, une couche de conductivité moyenne, au sein de l'interface. Lorsque l'interface oscille de part et d'autre de l'extrémité de la sonde, il en résulte un signal de déséquilibre d'un pont de Kohlrausch, dont on mesure la période. L'utilisation de deux compteurs chronomètres permet de mesurer les périodes propres consécutives.

\section{Influence de la rotation}

Rappelons des résultats publiés récemment [1]. L'expérience montre que la période propre d'une seiche d'interface en bassin rectangulaire augmente avec la rotation. Cette propriété est déjà connue dans le cas des seiches de surface (cf. G. I. Taylor, Rayleigh, Proudman, Lamb, A. Defant, D. B. Rao, ...). On trouve, au contraire, en milieu infini, une diminution de la période en fonction de la vitesse de rotation.

L'augmentation est faible mais parfaitement mesurable: ainsi une vitesse de rotation de 1 tour en 63 secondes augmente la période propre de $1 \%$ (cf. figure 4).

Il subsiste, toutefois, un écart systématique entre les périodes mesurées et celles calculées à partir de l'équation de dispersion (cf. $\S \mathrm{II}$ ), voisin de $0,6 \mathrm{~s}$. Celui-ci est attribué à l'état diffus de l'interface.

\section{Influence de la diffusion}

En effet, les liquides étant miscibles, la répartition verticale des densités ne prend pas la forme d'une fonction échelon $\left(\rho_{1}, \rho_{2}\right)$ comme le suppose notre théorie. L'interface a une certaine «épaisseur » qui augmente dans le temps, sous l'effet du cisaillement alternatif.

Même en début d'expérience, après le remplissage, l'épaisseur est de l'ordre du centimètre. Des mesures de période propre en fonction de l'épaisseur de l'interface ont permis, par extrapolation, de montrer qu'une interface idéale devait avoir une période propre inférieure de $0,55 \mathrm{~s}$ à celle qui est mesurée au cours d'une expérience habituelle (cf. figure 5).

Cette valeur est à rapprocher des $0,6 s$ signalés précédemment.

Il est donc raisonnable d'attribuer l'écart entre théorie et mesures à l'état diffus de l'interface.

\section{IV.2 - Configuration de l'onde}

A l'aide des suiveurs d'interface, on mesure les dénivellations instantanées de l'interface en une cinquantaine de points situés dans un quart du canal (cf. figure 6). Toutes les composantes élémentaires de l'onde sont en effet soit symétriques, soit antisymétriques par rapport au centre de l'interface.

Comme la diffusion augmente la période propre de l'onde, que la période d'excitation du canal est fixée une fois pour toutes, après que la résonance ait été atteinte (généralement en 30 ou 40 cycles), l'onde voit son amplitude diminuer sous l'effet de la diffusion à l'interface. Etant donné que les enregistrements s'effectuent pendant une cinquantaine de périodes, il convient de corriger chaque mesure par un coefficient lui restituant la valeur qu'elle aurait eue en l'absence de diffusion. Un procédé de correction a été mis au point à partir des informations fournies par un suiveur d'interface placé en un point fixe du canal et donne entière satisfaction.

L'analyse harmonique des enregistrements fournit alors les amplitudes maximales et les phases par rapport à une origine fixée de l'onde de fréquence fondamentale et des composantes harmoniques. Ces résultats permettent de 


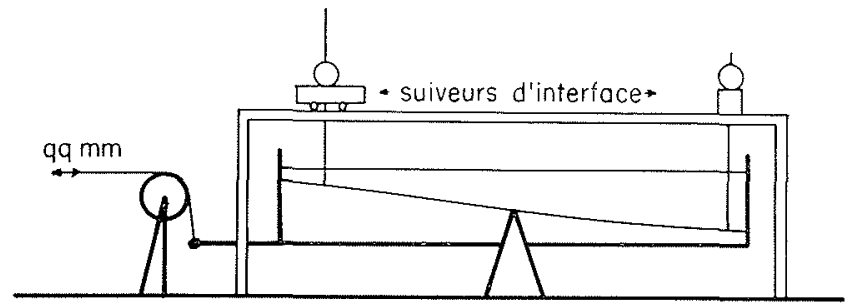

2/ Schéma de l'installation.
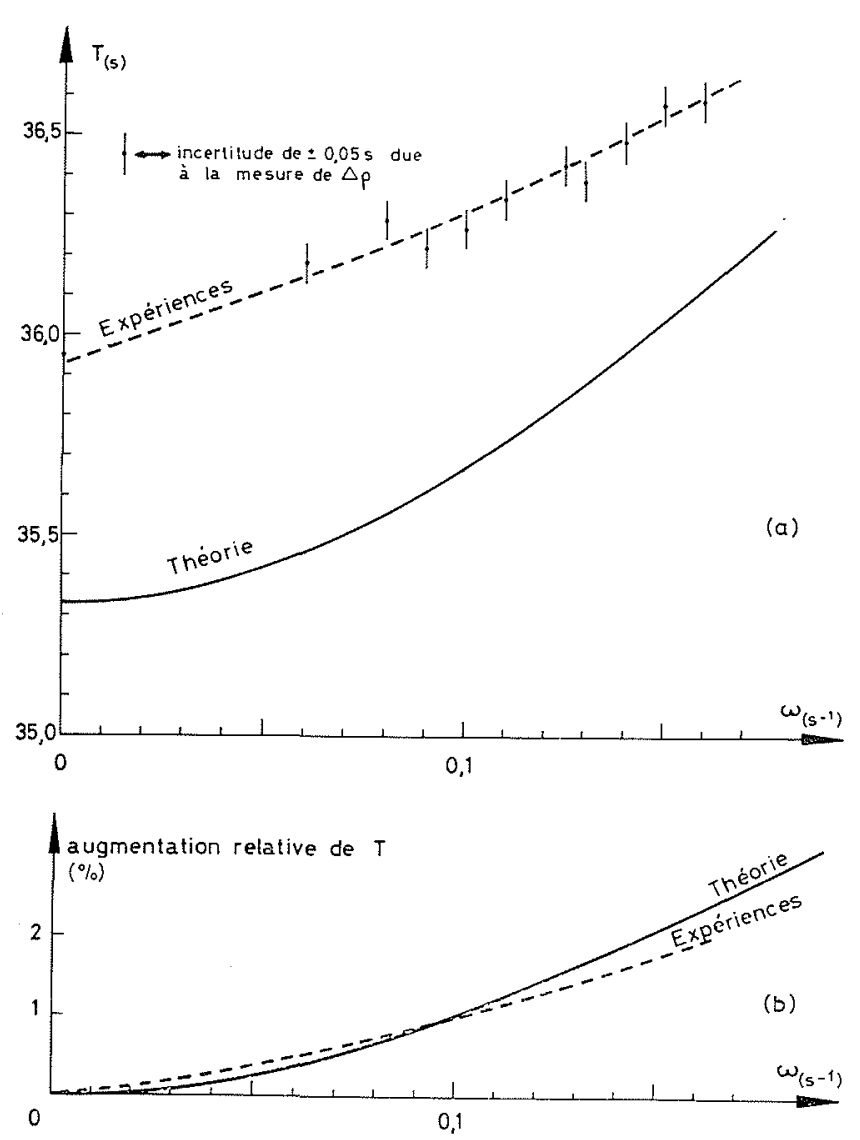

4/ Augmentation, par la rolation,

de la période propre de la seiche d'interface, pour $p_{1}=0,99895$ et $p_{2}=1,01745 \mathrm{~g} / \mathrm{cm}^{3}$.

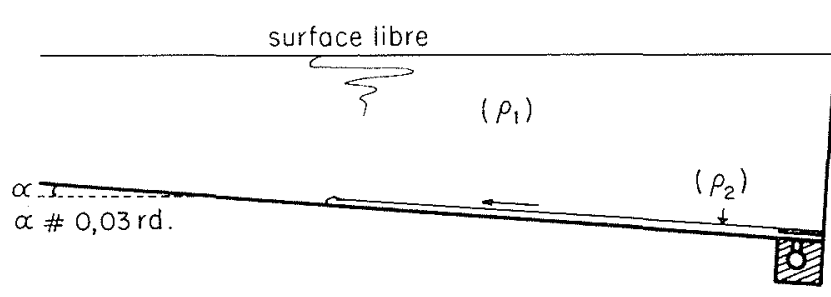

3/ Principe de la réalisation des bicouches.

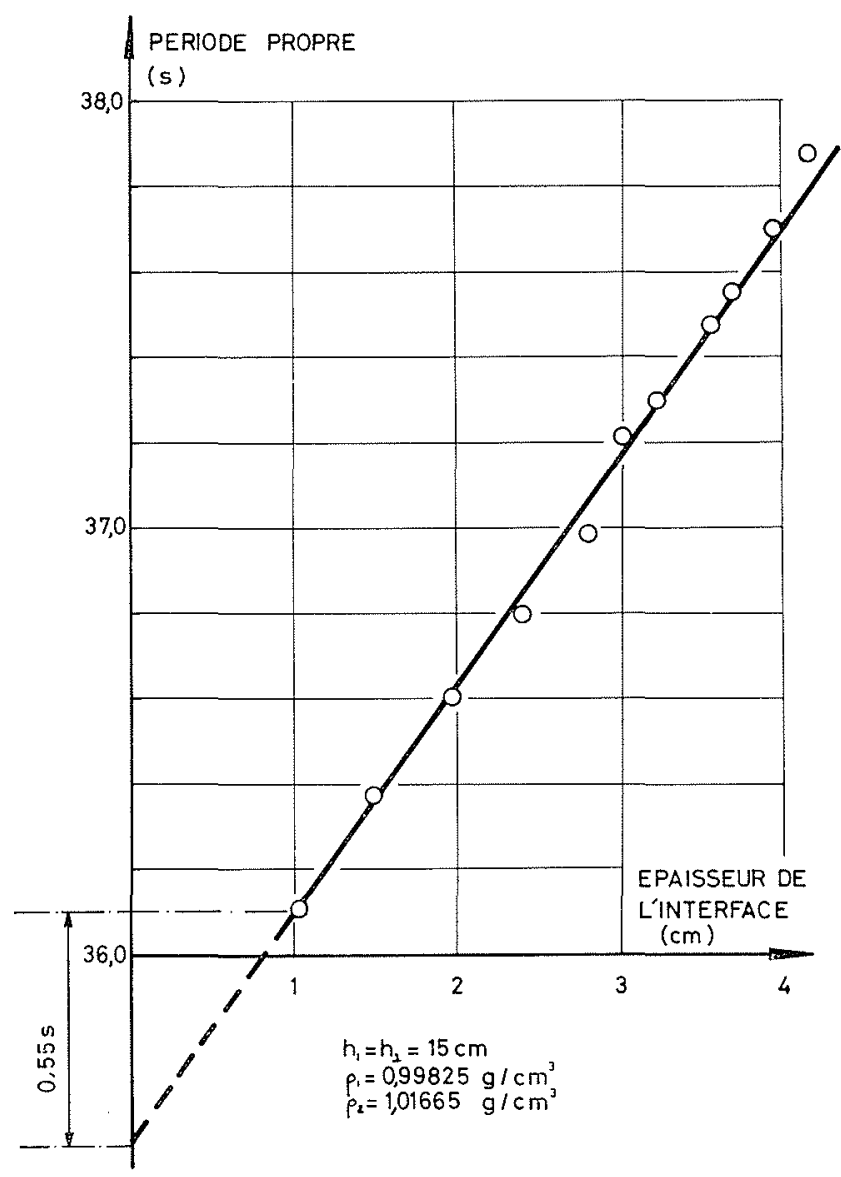

5/ Evolution de la période propre de la seiche interne en fonction de l'épaisseur de l'interface.

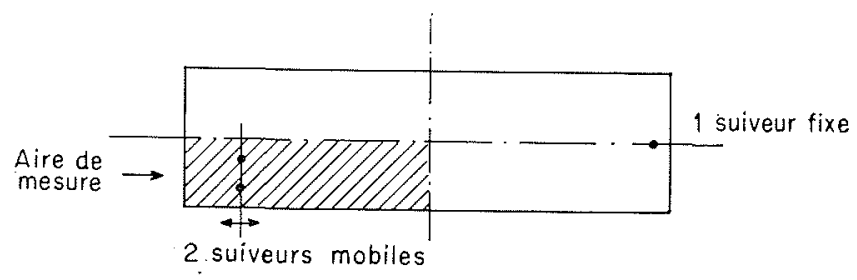

6/ Disposition des appareils de mesure. 


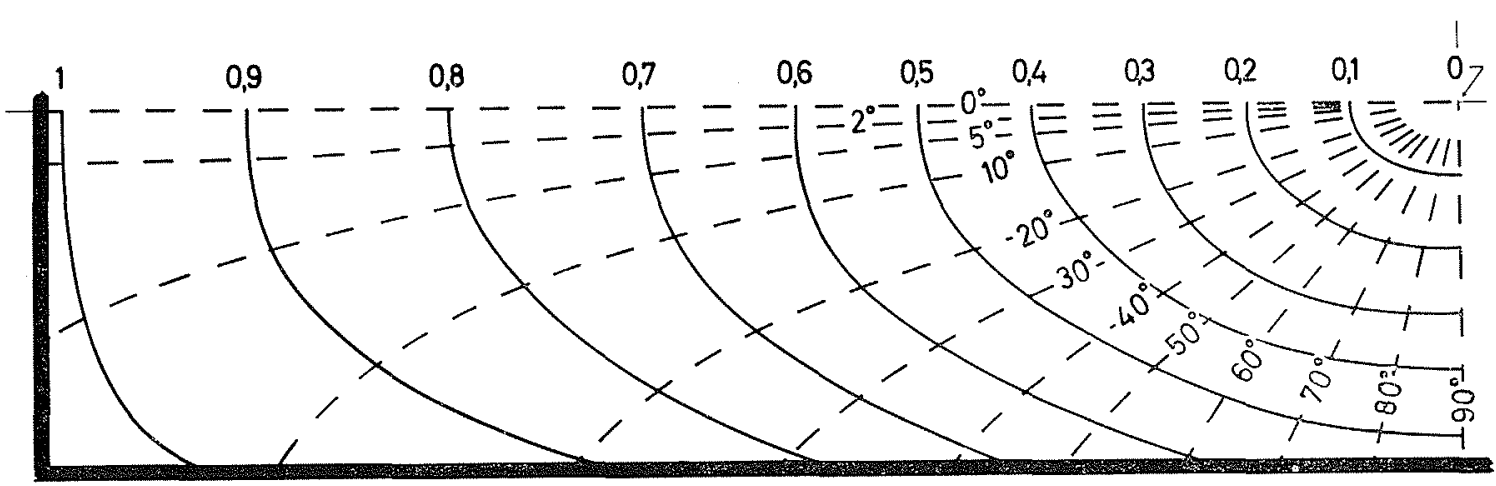

$\omega=0,1247 \mathrm{~s}^{-1}$

lignes d'égales amplitudes ; $\quad$ - - lignes d'égales phases

(résultats expérimentaux).

7/

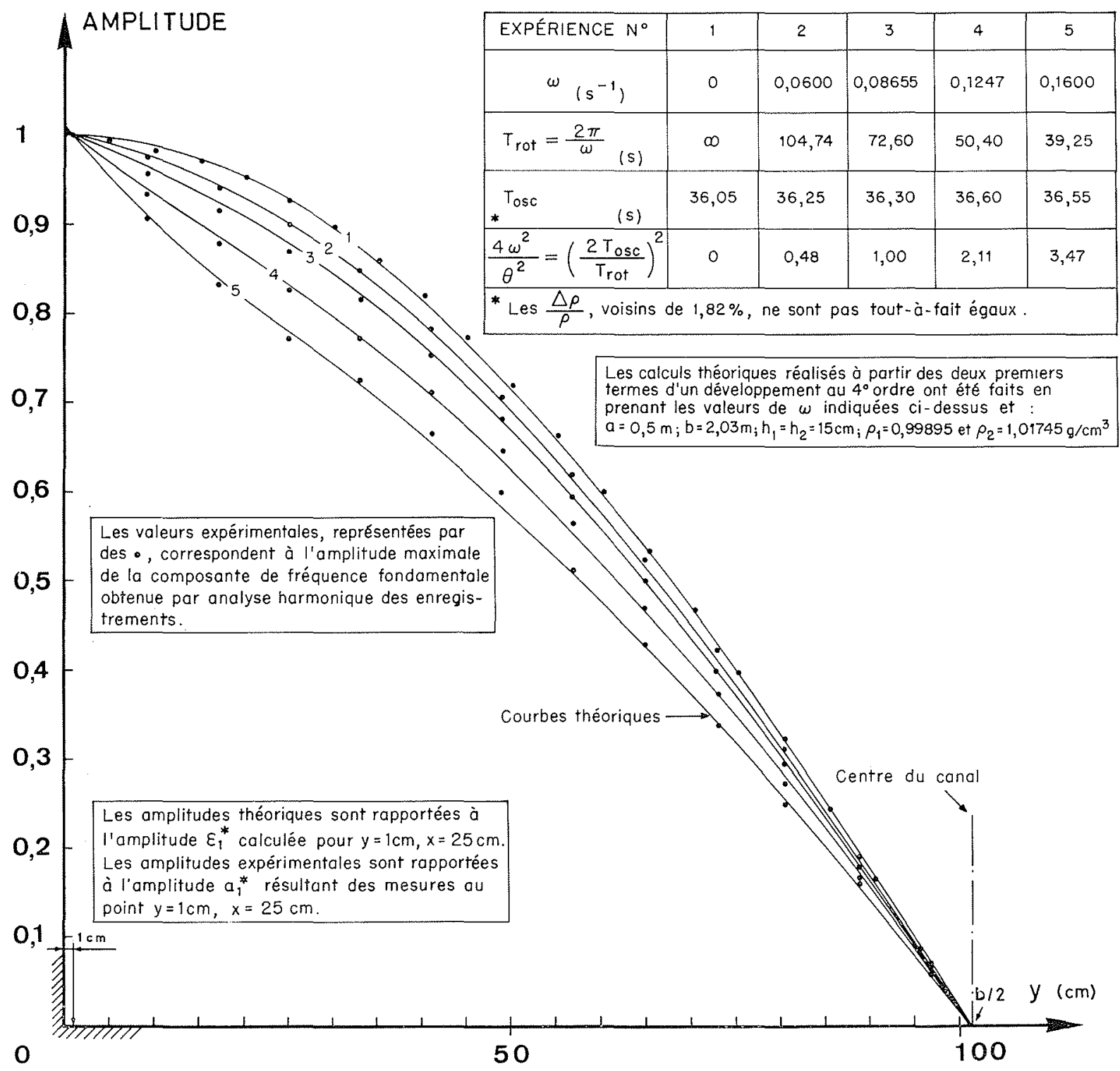

8/ Onde d'interface dans un bassin rectangulaire tournant:

profils d'amplitude, sur l'axe longitudinal médian, pour diverses vitesses de rotation. 
tracer les réseaux d'égales amplitudes et d'égales phases avec une précision moyenne de, respectivement, quelques pour mille et de quelques dixièmes de degrés. En fait, nous disposons d'un appareil très précis qui mesure un phénomène qui n'est pas parfaitement pur.

Intéressons-nous à la composante fondamentale.

Les réseaux ont une allure proche de celle d'une amphidromie de Kelvin dans la région centrale du canal mais se déforment au fur et à mesure que l'on s'en éloigne, sous l'influence des effets de parois qui font naître des ondes de Poincaré.

Les lignes d'égal marnage, parallèles au petit côté du bassin lorsque $\omega=0$, ont une allure globale d'ellipses allongées transversalement lorsque $\theta^{2}>4 \omega^{2}$, sont circulaires lorsque $\theta^{2}=4 \omega^{2}$, et ont une allure globale d'ellipses allongées longitudinalement lorsque $\theta^{2}<4 \omega^{2}$. En effet, on peut montrer qu'au quatrième ordre près, les lignes d'égal marnage d'une amplitude de Kelvin, ont pour équation, au voisinage du point amphidromique :

$$
\frac{x^{2}}{\theta^{2}}+\frac{y^{2}}{4 \omega^{2}}=\text { constante }
$$

Tous ces résultats feront l'objet d'une prochaine publication. Nous ne donnons ici que les réseaux obtenus pour la vitesse $\omega=0,1247 s^{-1}$ (cf. figure 7). Les amplitudes sont rapportées à l'amplitude déterminée sur l'axe longitudinal du canal à $1 \mathrm{~cm}$ de la paroi. L'origine des phases a été choisie sur le demi-axe longitudinal.

\section{IV.3 - Profils longitudinaux médians : exemple de comparaison théorie-expérience}

Pour comparer théorie et expériences, il est plus aisé de raisonner sur des profils d'ondes que sur des réseaux. Choisissons par exemple les profils longitudinaux médians, qui représentent des coupes réelles de l'onde en position extrême, puisque l'axe longitudinal du canal est une ligne équiphase.

Précisons que les valeurs expérimentales correspondent à l'amplitude maximale de la composante de fréquence fondamentale, obtenue par analyse harmonique des enregistrements et que les courbes théoriques ont été tracées à partir d'une expression constituée par les deux premiers termes d'un développement en série à l'ordre 4 (cf. $\S$ II).

On constate (cf. figure 8) que la concordance entre les résultats expérimentaux et les calculs théoriques approchés est très satisfaisante et que l'écart relatif moyen est de l'ordre de quelques pour mille.

Néanmoins, dans une région située à $50 \mathrm{~cm}$ de la paroi, l'écart peut atteindre 2 ou $3 \%$, lorsque la vitesse de rotation est relativement grande (expériences 4 et 5).

On peut attribuer celui-ci, soit au caractère approché de nos calculs (nous travaillons actuellement à leur amélioration), soit à la présence de termes spatiaux non-linéaires qui sont peut-être des termes en $\cos 2 k y$ comme le suggère le fait qu'il y ait un écart maximum à une distance de la paroi égale au quart de la longueur du canal, cf. [6].

Quoi qu'il en soit, l'écart est systématique et l'allure régulière des courbes expérimentales montre que la résolution des suiveurs d'interface, compte tenu des techniques de dépouillement, est pratiquement meilleure que le $1 / 10 \mathrm{~mm}$, pour des ondes de 3 à $4 \mathrm{~cm}$ d'amplitude.

\section{IV.4 - Ordre de grandeur des termes non-linéaires}

Il importe de se placer dans des conditions telles que les termes non linéaires soient faibles car nous ne possédons pas de méthode pour séparer, dans les enregistrements, les effets dus à la rotation que nous voulons étudier, et d'autres considérés alors comme «parasites», comme les termes non linéaires.

On trouve dans Thorpe [5], un calcul au deuxième ordre relatif aux oscillations libres d'une bicouche (liquides parfaits), en milieu infini (horizontalement): l'amplitude de l'interface est définie par:

$$
z=a \sin \theta t \cos k y+a^{2} k\left(z_{1}+z_{2} \cos 2 \theta t\right) \cos 2 k y(*)
$$

On montre que $z_{2}$ s'annule pour une valeur de $h_{1} / h_{2}$ très proche de 1 , ce qui justifie le choix de $h_{1}=h_{2}$ pour nos expériences en milieu tournant. Des expériences ont été réalisées en milieu non tournant pour $h_{1}=h_{2}$ afin d'évaluer l'ordre de grandeur des termes non-linéaires: le terme de fréquence fondamentale a une allure spatiale très proche d'un cos $k y$ (cf. courbe 1 de la figure 8 ); il subsiste en fait un écart systématique de $1 \%$ en moyenne, que l'on peut attribuer à des termes d'ordre supérieur comme $\cos 2 k y, \cos 3 k y, \cos 4 k y \ldots$

Il est probable que l'ordre de grandeur de ces effets non-linéaires est conservé lorsque l'on se place en système tournant; les vitesses de rotation ne dépassant pas, en effet, $0,16 s^{-1}$.

Par contre, si l'on veut justement étudier les effets nonlinéaires, l'expérience montre, ce que le calcul laissait prévoir, que l'on a intérêt à considérer des bicouches telles que $h_{1} / h_{2}$ soit assez grand.

\section{V - Conclusion}

La finesse de mesures et la technique employée pour leur dépouillement a permis de montrer la concordance des prévisions théoriques et des résultats expérimentaux avec une précision rarement atteinte en mécanique des fluides.

\section{Bibliographie}

[1] Suberville (J.-L.). - C.R. Acad. Sc. Paris, t. 277 (2 juillet 1973).

[2] TAYlor (G. I.). - Lond. Math. Soc., 2o série, 20, 1921, pp. 148-181.

[3] R^o (D. B.). - J. Fluid. Mech., 25, 1966, part 3, pp. 523-555.

[4] Bonnefille (R.). - Thèse d'état (Grenoble, 1968): «Contribution théorique et expérimentale à l'étude du régime des marées 》.

[5] Thorpe (S. A.). - J. Fluid Mech., 32, 1968, part 3, pp. 489-528.

[6] Suberville (J.-L.). - Thèse de Docteur-Ingénieur (Grenoble, 18-12-1974): «Ondes internes en fluide tournant. Contribution théorique et expérimentale ».

(*) a ne doit évidemment pas être confondu avec une notation utilisée précédemment, désignant la largeur de notre canal. 


\section{Discussion}

Président : M. le Professeur H. Lacombe

M. le Président remercie M. Suberville de son passionnant exposé et du film très suggestif qui l'a suivi; tout à fait dans la ligne des travaux de l'Institut de Mécanique de Grenoble, ils montrent comment, par des méthodes d'expérimentation très fines, on peut souvent contrôler les résultats du calcul par ceux de l'expérience.

Il ouvre ensuite la discussion :

M. CRÉPon intervient en ces termes :

Vous avez dit que, lorsqu'il y avait rotation, il y avait augmentation de la période propre; que devient la longueur d'onde?

M. Suberville répond :

En milieu tournant illimité, il est possible de définir des longueurs d'ondes en recherchant des solutions particulières correspondant à des ondes se propageant le long d'une direction donnée. Les deux premiers exemples qui viennent à l'esprit sont l'onde de Kelvin et l'onde à crête horizontale. L'existence de ces solutions est alors liée à la condition $\theta^{2}-4 \omega^{2}>0$, dans le cadre des théories classiques d'ondes longues. Ceci pose le problème des latitudes critiques.

Dans un bassin confiné, il en est tout autrement. Dans le cas que nous avons traité (bassin rectangulaire), la solution est recherchée sous la forme d'une combinaison linéaire d'ondes de Kelvin et de Poincaré, ayant chacune leurs caractères propres (par exemple, la période propre des ondes de Kelvin ne dépend pas de la rotation), mais dont la superposition conduit à un résultat opposé au cas du milieu illimité : les ondes se propagent, quel que soit le signe de $\theta^{2}-4 \omega^{2}$. Il est encore possible de définir une longucur d'onde; celle-ci est proportionnelle à la période propre $T$ du système; on a en effet :

$$
\lambda=\left[\mathrm{g} \frac{\Delta \varrho}{\varrho} \frac{h_{1} h_{2}}{h_{1}+h_{2}}\right]^{1 / 2} . \mathrm{T} .
$$

La quantité $\lambda / T$, qui n'est autre que la célérité de l'onde est indépendante de la vitesse de rotation $\omega$, si la pression est hydrostatique. Le paramètre $\lambda$ tend vers le double de la longueur du canal lorsque $\omega$ tend vers zéro.

La période $T$, disions-nous, augmente avec la rotation; or, un calcul analogue, dans le cas d'une baie rectangulaire (domaine semiinfini) aboutit au résultat contraire. Ceci est étonnant.

D'autre part, on peut montrer, en s'écartant des hypothèses restrictives des théories d'ondes longues, que l'introduction des accélérations verticales permet de concevoir deux types d'ondes : les premières (I), tendant à la limite vers des ondes longues, les secondes (II) possédant un ou plusieurs plans nodaux horizontaux.

Dans le cas d'un bassin rectangulaire, on peut définir ce que nous appelerons, cette fois, une psendo-longueur d'onde $\lambda^{\prime}$, liée à la dimension verticale du système. La quantité $\lambda^{\prime} / T$ est alors une fonction de $\omega$, faiblement croissante dans des conditions naturelles.

Ces propriétés sont valables aussi bien pour les ondes de surface que pour les ondes dinterface. Reportons-nous aux résultats que nous avons obtenus à propos des périodes propres à l'onde d'inter face (type I) en milieu tournant. L'écart entre théorie et expériences est de l'ordre de $0,5 \mathrm{~s}$ en moyenne. Il aurait été de $0,8 \mathrm{~s}$ si la prassion avait été supposée hydrostatique, soit un écart $60 \%$ supérieur.

Les ondes de type II modifient les données du problème relatif aux latitudes critiques; elles se propagent le long d'une direction donnée lorsque $\theta^{2}-4()^{2}<0$.

A ce propos, Fultz, en 1959, écrivait que l'importance de ces ondes était à redécouvrir, ce qui est confirmé par les travaux de Saint-Guily (1962).

M. le Président clôt la discussion et donne la parole à M. CRÉpon pour l'exposé de sa communication. 